\title{
Communication
}

\section{Localization of MLH3 at the Centrosomes}

\section{Lennart M. Roesner ${ }^{1,2, *}$, Christian Mielke ${ }^{3}$, Silke Faehnrich ${ }^{1}$, Yvonne Merkhoffer ${ }^{1}$,} Kurt E. J. Dittmar ${ }^{4}$, Hans G. Drexler ${ }^{1}$ and Wilhelm G. Dirks ${ }^{1}$

1 Leibniz-Institute DSMZ-German Collection of Microorganisms and Cell Cultures, Department of Human and Animal Cell Lines, Braunschweig 38124, Germany; E-Mails: sif@dsmz.de (S.F.); yvonne.merkhoffer@dsmz.de (Y.M.); hdr@dsmz.de (H.G.D.); wdi@dsmz.de (W.G.D.)

2 Hannover Medical School, Division of Immunodermatology and Allergy Research, Department of Dermatology and Allergy, Hannover 30625, Germany

3 Institute of Clinical Chemistry and Laboratory Diagnostics, Heinrich-Heine-University, Medical School, Düsseldorf 40225, Germany; E-Mail: christian.mielke@med.uni-duesseldorf.de

4 Helmholtz Centre of Infection Research, Braunschweig 38124, Germany;

E-Mail: ked@helmholtz-hzi.de

* Author to whom correspondence should be addressed; E-Mail: roesner.lennart@mh-hannover.de; Tel.: +49-511-532-5054; Fax: +49-511-532-8111.

Received: 10 June 2014; in revised form: 7 July 2014 / Accepted: 4 August 2014 / Published: 11 August 2014

\begin{abstract}
Mutations in human DNA mismatch repair (MMR) genes are commonly associated with hereditary nonpolyposis colorectal cancer (HNPCC). MLH1 protein heterodimerizes with PMS2, PMS1, and MLH3 to form MutL $\alpha$, MutL $\beta$, and MutL $\gamma$, respectively. We reported recently stable expression of GFP-linked MLH3 in human cell lines. Monitoring these cell lines during the cell cycle using live cell imaging combined with confocal microscopy, we detected accumulation of MLH3 at the centrosomes. Fluorescence recovery after photobleaching (FRAP) revealed high mobility and fast exchange rates at the centrosomes as it has been reported for other DNA repair proteins. MLH3 may have a role in combination with other repair proteins in the control of centrosome numbers.
\end{abstract}

Keywords: DNA mismatch repair (MMR); $M L H 3$; centrosome 


\section{Introduction}

Mutations in human DNA mismatch repair (MMR) genes are commonly associated with hereditary nonpolyposis colorectal cancer (Lynch syndrome). To form the MMR protein complexes, MSH2 heterodimerizes with MSH3 and MSH6, while MLH1 heterodimerizes with PMS2, PMS1, and MLH3 to form MutS $\alpha$, MutS $\beta$, MutL $\alpha$, MutL $\beta$, and MutL $\gamma$, respectively. While MutL $\beta$ is the key player in MMR, reports on the function of MutL $\gamma$ concentrate on observations in meiotic recombination events. Knockout-mice revealed a crucial role of MutL $\gamma$ in crossing-over during meiosis [1-4], but investigating human MutL $\gamma$ was hampered by a lack of appropriate and accessible experimental systems. Endogenous $M L H 3$ expression is hardly detectable in human cells since being expressed up to 60 times lower than other MMR proteins [5] and stable recombinant expression could be shown to interfere with cell physiology and is reported to be toxic to human cell lines $[5,6]$. Recently, we reported for the first time stable expression of recombinant green fluorescent protein (GFP)-linked MLH3 in human cell line [7]. Using multicistronic vector systems, gene expression was observed to be tolerated if quenched to a low level. Biological function of recombinant MLH3 was confirmed by exclusive nuclear localization, the ability to bind endogenous partner proteins and a fast recruitment to sites of UV laser-induced DNA damage. To investigate the behaviour of MLH3 in the cell, the stable transfected cell lines were monitored during the cell cycle using live cell imaging combined with confocal microscopy.

\section{Results and Discussion}

We detected accumulation of GFP-MLH3 forming two distinct fluorescent foci per cell. The position of the two foci on each side of the aequatorial plane during the different phases of the cell cycle suggested that these might resemble accumulation of MLH3 at the centrosomes (Figure 1a). Immunostaining using an antibody against the centrosome-associated $\gamma$-Tubulin (Sigma-Aldrich, St. Louis, MO, USA) confirmed this hypothesis by co-localization with GFP-MLH3-foci (Figure 1b).

To investigate the nature of these accumulation foci, fluorescence recovery after photobleaching (FRAP) technique was applied. After photobleaching of one of two foci (compare "before" to " $0 \mathrm{~s}$ "-picture), every $4 \mathrm{~s}$ an image was taken. Recovery of signal relative to the other centrosomal foci is achieved within $28 \mathrm{~s}$. Fluorescence recovery to $100 \%$ was observed in eight independent measurements (Figure 2). This high mobility resembles fast exchange rates at the centrosomes, which has been reported also for other DNA repair proteins $[8,9]$.

By taking advantage of the capacity of confocal microscopy to eliminate any reflected light from out of the focus, we were able to detect structures within the cell, that are invisible in epifluorescence microscopes. Of course, recombinant expression always harbours the danger of producing artefacts by vast over-expression. In our hands, we achieved a remarkably low expression level applying a multicistronic expression system [7], which turned out to bypass the described toxicity of overexpressed MLH3 [5,6]. Furthermore, the centrosome-associated localization could only be observed for GFP-MLH3, not GFP-MLH1, GFP-PMS1, or GFP-PMS2, where even higher expression levels are achieved by exogenous expression (data not shown). Without the possibility to stain 
endogenous MLH3 due to the lack of suitable antibodies, we therefore regard our expression system as the best model currently available to study the cellular localization of MLH3.

Figure 1. MLH3 accumulates at the centrosomes. (A) Confocal microscopy of stably transfected cells (cell line 293), in different stages of mitosis as indicated. GFP-MLH3 accumulates at two foci per cell. Exemplary pictures were chosen, where both fluorescent foci were detected in the same focal pane; and (B) Immunostaining of formalin-fixed and permeabilized stably transfected cells using anti- $\gamma$-tubulin antibody and a secondary antibody coupled to tetramethylrhodamine.

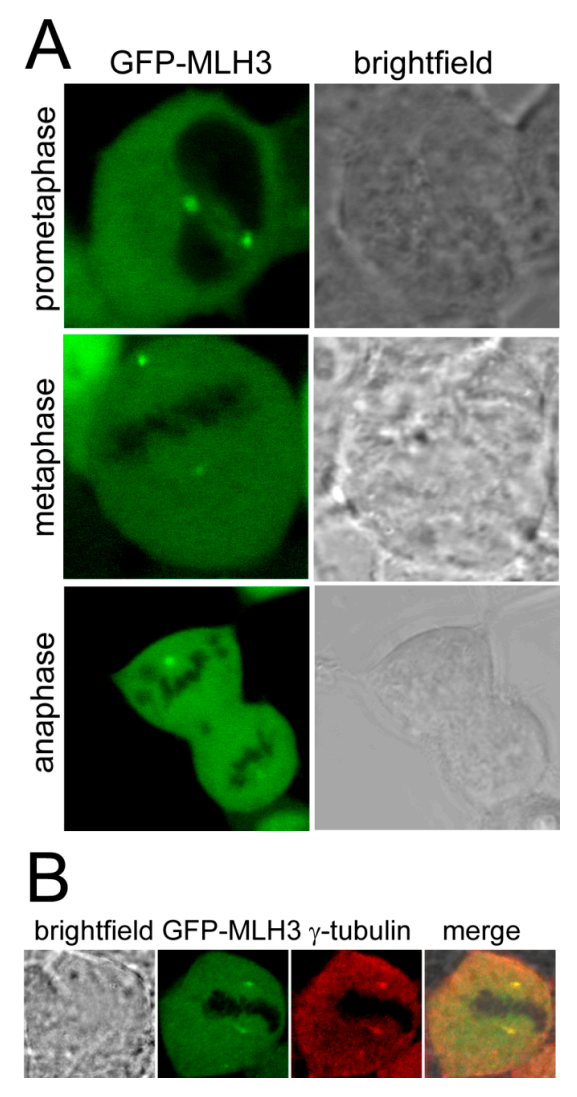

However, a potential function of MLH3 at the centrosomes may lie in the control of centrosome numbers. If not under proper control, a multipolar spindle apparatus, due to supernumerary centrosomes, leads to a defective mitosis and is described for a number of different cancer types [10]. Regulation of centrosome number is most probably achieved by several spindle assembly checkpoints [11], where repair proteins can trigger a cell cycle arrest in case of errors. Up to now, a number of repair proteins of the homologous recombination and non-homologous end joining-pathways have been found to be involved [12]. However, also for the MMR, key player MSH2 functions in centrosome maintenance are reported [13]; $\mathrm{Msh}^{-1-}$ mice showed an increased number of centrosomes in every third cell as well as modifications at the telomers. The localization of $\mathrm{MSH} 2$ at the centrosomes was reported subsequently by Narine et al., 2010 [14]. Furthermore, MMR family member MSH4 may be associated to centrosomes, as binding to the microtubule-synthesis associated protein VBP1 (von Hippel-Lindau binding protein 1) has been shown [15,16]. Since MLH3 harbours a binding site to MSH4 [4], this might be the link attracting the protein to the centrosomes during mitosis and an interesting target for further investigation. 
Figure 2. Fluorescence recovery after photobleaching (FRAP) of centrosomal GFP-MLH3 foci. Data shown represents eight independent measurements. Error bars represent the standard error of the mean.

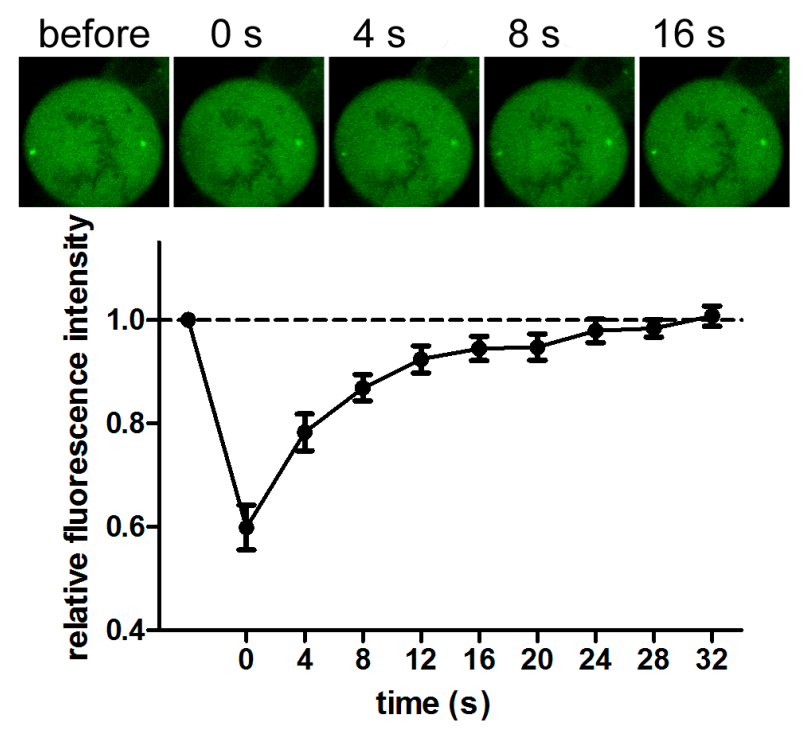

\section{Experimental Section}

\subsection{Cell Lines}

Human embryonal kidney cell line 293 (DSMZ, Braunschweig, Germany, ACC 305) was grown in 90\% Dulbecco's MEM supplemented with $10 \%$ fetal bovine serum, $100 \mathrm{U} / \mathrm{mL}$ penicillin, $100 \mu \mathrm{g} / \mathrm{mL}$ streptomycin and Glutamax-I (Invitrogen, Karlsruhe, Germany) at $37{ }^{\circ} \mathrm{C}$ with $10 \% \mathrm{CO}_{2}$. Subculturing was achieved by splitting confluent cultures about 1:5 every 3 days by detaching cells by tapping the culture flasks. Stable expressing cell lines were generated as described before [7]. Briefly, MLH3 was amplified by RT-PCR from primary human MRC-5 lung cells. cDNAs were fused $N$-terminally and in frame to GFP and $C$-terminally to an internal ribosomal entry site (IRES), while latter one was fused to the selectable marker gene puromycin- $N$-acetyltransferase to be expressed in a bi- or tricistronic manner. Cells were transfected with plasmid DNA using SuperFect Transfection Reagent according the manufacturer's guideline (Qiagen, Hilden, Germany). Forty-eight hours after transfection, selective pressure was applied by $0.7 \mu \mathrm{g} / \mathrm{mL}$ puromycin. After 14 to 20 days clones were isolated, expanded and analysed for fluorescence and exclusive nuclear localization.

\subsection{Immunofluorescence Staining}

Cells were grown in chamberslides (Thermo Scientic Nunc LabTek, Waltham, MA, USA) upon semi-conluency and were fixed subsequently using $4 \%$ formaldehyde/PBS for $15 \mathrm{~min}$ at $37{ }^{\circ} \mathrm{C}$. After permeabilization over night in $0.1 \mathrm{M}$ Tris- $\mathrm{HCl} / 50 \mathrm{mM}$ EDTA/0.5\% TritonX-100 blocking was performed for $2 \mathrm{~h}$ with $3 \%$ bovine serum albumin $/ 0.1 \%$ Tween 20 . Antibodies were applied in PBS/1\% fetal bovine serum $/ 0.1 \%$ Tween 20 for $2 \mathrm{~h}$ each. Mouse monoclonal anti- $\gamma$-tubulin antibody (Sigma-Aldrich, St. Louis, MO, USA) was applied 1:50, followed by goat-anti-mouse-tetramethylrhodamine (Southern Biotechnology, Birmingham, AL, USA) 1:200. 


\subsection{Microscopy}

Microscopy was performed on a Zeiss LSM 510 Meta (Zeiss, Jena, Germany) using the $488 \mathrm{~nm}$ laser line to excite GFP and a Plan-NeoFluar $40 \times / 1.3$ oil immersion objective. The pinhole was set to $1-2 \mathrm{~mm}$. Live cell imaging experiments were performed at physiological conditions in chamberslides, using a heating stage in combination with an incubation unit and an active gas mixer (Ibidi, Martinsried, Germany) to achieve $37{ }^{\circ} \mathrm{C}$ temperature and physiologic $\mathrm{CO}_{2}$-content. Furthermore, an objective heater (Zeiss) excluded any undesired cooling by the objective itself.

\subsection{Fluorescence Recovery after Photobleaching (FRAP)}

FRAP was carried out by bleaching a defined area inside the nucleus with $25 \%$ laser power, $100 \%$ transition and 20 iterations. Several images were taken before bleaching, followed by one image directly after the bleach and consecutive images every $2 \mathrm{~s}$ with $5 \%$ transition. Fluorescence intensity was measured at each time point. Background was subtracted before fluorescence loss due to monitor bleaching was normalized, to calculate relative intensity $I_{\text {rel }}=\left(T_{0} \times I_{\mathrm{t}}\right) /\left(T_{\mathrm{t}} \times I_{0}\right)$, where $T$ is the total cellular intensity at the time points 0 and $\mathrm{t}$ and $I$ the average intensity of the bleached region at the time points 0 and $\mathrm{t}$.

\section{Conclusions}

MMR protein $M L H 3$ accumulates with fast exchange rates at the centrosomes.

\section{Author Contributions}

L.M.R. and W.G.D. performed the experiments and wrote the manuscript; S.F. and Y.M. performed experiments; C.M., K.E.D. and H.G.D. provided technical help and/or material and contributed to the manuscript.

\section{Conflicts of Interest}

The authors declare no conflict of interest.

\section{References}

1. Cohen, P.E.; Pollack, S.E.; Pollard, J.W. Genetic analysis of chromosome pairing, recombination, and cell cycle control during first meiotic prophase in mammals. Endocr. Rev. 2006, 27, 398-426.

2. Kolas, N.K.; Svetlanov, A.; Lenzi, M.L.; Macaluso, F.P.; Lipkin, S.M.; Liskay, R.M.; Greally, J.; Edelmann, W.; Cohen, P.E. Localization of MMR proteins on meiotic chromosomes in mice indicates distinct functions during prophase I. J. Cell. Biol. 2005, 171, 447-458.

3. Lipkin, S.M.; Moens, P.B.; Wang, V.; Lenzi, M.; Shanmugarajah, D.; Gilgeous, A.; Thomas, J.; Cheng, J.; Touchman, J.W.; Green, E.D.; et al. Meiotic arrest and aneuploidy in MLH3-deficient mice. Nat. Genet. 2002, 31, 385-390. 
4. Santucci-Darmanin, S.; Neyton, S.; Lespinasse, F.; Saunieres, A.; Gaudray, P.; Paquis-Flucklinger, V. The DNA mismatch-repair MLH3 protein interacts with MSH4 in meiotic cells, supporting a role for this MutL homolog in mammalian meiotic recombination. Hum. Mol. Genet. 2002, 11, 1697-1706.

5. Charbonneau, N.; Amunugama, R.; Schmutte, C.; Yoder, K.; Fishel, R. Evidence that hMLH3 functions primarily in meiosis and in hMSH2-hMSH3 mismatch repair. Cancer Biol. Ther. 2009, $8,1411-1420$.

6. $\quad$ Lipkin, S.M.; Wang, V.; Jacoby, R.; Banerjee-Basu, S.; Baxevanis, A.D.; Lynch, H.T.; Elliott, R.M.; Collins, F.S. MLH3: A DNA mismatch repair gene associated with mammalian microsatellite instability. Nat. Genet. 2000, 24, 27-35.

7. Roesner, L.M.; Mielke, C.; Fahnrich, S.; Merkhoffer, Y.; Dittmar, K.E.; Drexler, H.G.; Dirks, W.G. Stable expression of MutL $\gamma$ in human cells reveals no specific response to mismatched DNA, but distinct recruitment to damage sites. J. Cell. Biochem. 2013, 114, 2405-2414.

8. Mortusewicz, O.; Leonhardt, H. XRCC1 and PCNA are loading platforms with distinct kinetic properties and different capacities to respond to multiple DNA lesions. BMC Mol. Biol. 2007, 8, 81.

9. Mortusewicz, O.; Rothbauer, U.; Cardoso, M.C.; Leonhardt, H. Differential recruitment of DNA Ligase I and III to DNA repair sites. Nucleic Acids Res. 2006, 34, 3523-3532.

10. Nigg, E.A. Centrosome aberrations: Cause or consequence of cancer progression? Nat. Rev. Cancer 2002, 2, 815-825.

11. Kwon, M.; Godinho, S.A.; Chandhok, N.S.; Ganem, N.J.; Azioune, A.; Thery, M.; Pellman, D. Mechanisms to suppress multipolar divisions in cancer cells with extra centrosomes. Genes Dev. 2008, 22, 2189-2203.

12. Shimada, M.; Komatsu, K. Emerging connection between centrosome and DNA repair machinery. J. Radiat. Res. 2009, 50, 295-301.

13. Campbell, M.R.; Wang, Y.; Andrew, S.E.; Liu, Y. MSH2 deficiency leads to chromosomal abnormalities, centrosome amplification, and telomere capping defect. Oncogene 2006, $25,2531-2536$.

14. Narine, K.A.; Keuling, A.M.; Gombos, R.; Tron, V.A.; Andrew, S.E.; Young, L.C. Defining the DNA mismatch repair-dependent apoptotic pathway in primary cells: Evidence for p53-independence and involvement of centrosomal caspase 2. DNA Repair 2010, 9, 161-168.

15. Geissler, S.; Siegers, K.; Schiebel, E. A novel protein complex promoting formation of functional $\alpha$ - and $\gamma$-tubulin. EMBO J. 1998, 17, 952-966.

16. Her, C.; Wu, X.; Griswold, M.D.; Zhou, F. Human MutS homologue MSH4 physically interacts with von Hippel-Lindau tumor suppressor-binding protein 1. Cancer Res. 2003, 63, 865-872.

(C) 2014 by the authors; licensee MDPI, Basel, Switzerland. This article is an open access article distributed under the terms and conditions of the Creative Commons Attribution license (http://creativecommons.org/licenses/by/3.0/). 